\title{
Characterization of Endophytic Microbial Communities in Store-Bought Kale Evaluated by Different Plant Tissue Homogenization Methods
}

\author{
C. Ruth McNees, Audrey D. Law, and Luke A. Moe ${ }^{\dagger}$ \\ Department of Plant and Soil Sciences, University of Kentucky, Lexington, KY 40546-0312
}

Accepted for publication 26 May 2020.

\section{ABSTRACT}

Endophytic microorganisms live in intercellular and vascular spaces of plants and span the continuum from beneficial to pathogenic in both plants and animals. Increasing human consumption of fruits and vegetables has placed an emphasis on identifying and studying those microbes that colonize the internal tissues of plants, with a goal of limiting populations of enteric pathogens in store-bought foods meant for raw consumption, such as leafy greens. Culture-independent (i.e., metagenomic) methods are increasingly used to obtain an accurate snapshot of plant microbial communities, yet technical hurdles limit the accuracy and throughput of these methods. This includes the low-throughput nature of plant tissue maceration, and the prevalence of plant plastid DNA in metagenomic DNA extracts, which is typically coamplified via PCR strategies that target the bacterial 16S rRNA gene. In this study, we use kale (Brassica oleracea) as a model to explore the leafy green endophytic microbiome and to compare how two tissue maceration techniques used in traditional endophyte research compare in culture-independent microbiome studies using the Illumina Miseq platform. Three different brands of store-bought kale were surface sterilized and subjected to two tissue maceration strategies: enzyme digestion and blender processing. Analysis of 16S rRNA gene amplicon libraries revealed two highly abundant operational taxonomic units present in all libraries, one classified to the genus Pseudomonas and one to the family Enterobacteriaceae. Community structure and membership were highly similar among brands and between tissue maceration strategies, suggesting that both enzyme digestion and blender processing are suitable methods. Nonetheless, enzyme digestion may increase sample throughput and minimize steps involved in sample processing.

Keywords: bacteriology, Brassica oleracea, endophytes, kale, microbiome
Bacterial endophytes play an important role in plant function, showing symbiotic relationships with host plants resulting in improved plant health and crop production through nutrient acquisition, pathogen resistance, removal of contaminants, phytohormone production, and inducing plant immune responses (Franco et al. 2007; Glick 2014; Ryan et al. 2008). While foliar bacterial endophytes typically show lower abundance and diversity than root endophytes, the leaf endosphere is known to harbor microbes both beneficial and pathogenic to plants and humans (Brandl 2006; Teplitski et al. 2009; Tyler and Triplett 2008), and these microbes play myriad roles in the postharvest quality of certain agronomic and horticultural crops (Law et al. 2016; McNees et al. 2019).

Technical challenges may limit research on foliar endophytes (McNees et al. 2019). Studies of foliar endophytic microbial

${ }^{\dagger}$ Corresponding author: luke.moe@uky.edu

The author(s) declare no conflict of interest.

(C) 2020 The American Phytopathological Society populations rely on surface sterilization of the leaf, followed by tissue maceration and either cultivation of microbes from macerate using solid medium, or DNA extraction of the macerate for cultureindependent analysis. Cultivation-based strategies have the benefit of generating microbial stocks that can be used for further characterization, but these strategies are likely to exclude low abundance microbes and those refractory to conventional cultivation. Cultivation-independent, or metagenomic, strategies can capture a larger portion of the population, resulting in a more accurate representation of the total microbial community. In general, indexed PCR primers targeting one or more hypervariable regions of the $16 \mathrm{~S}$ rRNA gene are used to generate amplicons for sequencing, with the resulting data used to assess community composition. The inherent difficultly of this approach lies in the optimization of microbial DNA extraction from plant tissue and minimizing amplicons resulting from plant plastid and mitochondrial rRNA genes. In root tissue or woody plant parts, this interference is less abundant. However, in fresh green leaves rich with chloroplasts, the coamplification of these sequences easily overwhelm the microbial community when no effort is made to reduce them. 
Several strategies for addressing these issues have been developed by researchers studying foliar endophytes, each with benefits and drawbacks. Adding locking nucleic acids or protein nucleic acids to the PCR to exclude amplification of plastid and mitochondrial DNA can be effective, but these specialized primers are expensive and must be designed for the specific host plant (Ikenaga and Sakai 2014; Sakai and Ikenaga 2013). Alternatively, primers can be used that target regions other than V4 of the 16S rRNA gene that will exclude mitochondrial or plastid DNA, such as 799F and 1492R primer set (V8-9); however, variable regions other than V4 can be more difficult to align and classify, and NGS analysis will result in more sequencing errors if the reads do not completely overlap (Beckers et al. 2016; Kozich et al. 2013; Schloss et al. 2009; Sun et al. 2008). Physically separating bacterial and plant cells before DNA extraction can successfully reduce plastid/ mitochondrial sequences by 80 to $90 \%$, but it is time consuming, limiting the number of samples that can be processed, and requires large amounts of leaf tissue (Ikeda et al. 2009, 2010; Jiao et al. 2006; Wang et al. 2008). All of these methods have been employed successfully to combat the problem of mitochondrial/ plastid DNA interference in culture-independent study of foliar endophytes, with the method chosen depending on the research goals and available resources of the investigator.

This paper is concerned with the physical separation of bacteria from leaf tissue, which is most applicable in the case where amplification of the V4 region is desirable, or necessary for comparison and/or continuity purposes, and the amount of plant tissue is not extremely limited. Two primary methods of tissue maceration to expose bacteria within the leaf can be found in the literature: either physical maceration by grinding or the use of a blender (blade or paddle type), or enzyme digestion of leaf tissue using a cellulose/ macerozyme solution to dissolve plant cell walls (Ikeda et al. 2009, 2010; Jiao et al. 2006; Prior et al. 2014; Wang et al. 2008). Either method may be preferable from a logistical standpoint, depending on the available equipment of the researcher, however it is unknown if these two methods differ with respect to culture-independent microbial community analysis. Studies that compared methods of sample processing and storage, DNA extraction, primer design, and PCR protocols have shown differences in diversity and community composition with culture-independent analysis, indicating that methods and experimental design must be carefully considered (Maropola et al. 2015; Sergeant et al. 2012).

Here we explore endophytic populations in commercially bought bundles of kale purchased from a Lexington, KY grocery outlet, employing both blender and enzyme methods of tissue maceration in order to determine if there are differences in diversity and community composition between the two methods. Kale is a leafy cultivar of the family Brassicaceae that is grown widely and valued for its status as a so-called superfood, owing to its nutrient- and vitamin-rich leaves, along with high concentrations of antioxidants.
Kale was chosen as a model for three reasons: it is most commonly consumed uncooked and therefore endophytic microbes are likely to be consumed, no literature exists on the kale endophytic microbiome, and the prominent waxy layer makes maceration more difficult than other leafy greens, meaning that a maceration strategy optimized for kale should function with other leafy greens as well.

\section{MATERIALS AND METHODS}

In this study, kale bunches were processed in the same manner with the exception of the method used to homogenize the leaf tissue (Fig. 1). Three leaves from each of three brands were surface sterilized in the following manner. In a sterile sample bag, each leaf was rinsed thoroughly in sterile water, followed by $100 \mathrm{ml}$ of $95 \%$ ethanol with gentle shaking for $5 \mathrm{~min}, 100 \mathrm{ml}$ of $0.5 \%$ bleach with gentle shaking for $2 \mathrm{~min}, 100 \mathrm{ml}$ of $70 \%$ ethanol with gentle shaking for $2 \mathrm{~min}$, and finally rinsed three times with sterile water, shaking gently $1 \mathrm{~min}$ each time. Surface sterilization was checked by applying $1 \mathrm{ml}$ of water from the last wash to nutrient agar plates. No growth was observed after 1 week of incubation at $28^{\circ} \mathrm{C}$. Leaves were then bisected aseptically along the midrib to produce two halves that were homogenized independently using either blender processing (BP) or enzyme digestion (ED). For blender processing, leaf tissue was added to a blender jar (Kinematica Microtron MB 550) with $400 \mathrm{ml}$ of BCE buffer (50 mM Tris-HCl, pH 7.5, 1\% Triton X-100, 2 mM 2-mercaptoethanol added directly before use), processed at full speed for $1 \mathrm{~min}$, and rested on ice for $1 \mathrm{~min}$, with last two steps repeated a total of three times (Ikeda et al. 2009, 2010). Blender jars were thoroughly cleaned and autoclaved between use. For enzyme digestion, leaf tissue was rolled and sliced into approximately $1-\mathrm{mm}$-thin strips using a sterile razor blade, and then immersed in enzyme solution ( $1.5 \%$ cellulase and $0.4 \%$ macerozyme R10 added directly before use to filter sterilized buffer, $0.4 \mathrm{M}$ mannitol, $20 \mathrm{mM} \mathrm{KCl}, 20 \mathrm{mM}$ MES, pH 5.7) overnight (18 h) at $25^{\circ} \mathrm{C}$ with gentle shaking $(50 \mathrm{rpm})$ in autoclaved 250 -ml flasks (Prior et al. 2014).

All homogenized samples were subjected to the same downstream process of differential centrifugation and density-gradient centrifugation employing Nycodenz (Accurate Chemical \& Scientific Corp., Westbury, NY) to separate the microbial cells from remaining plant tissue (Ikeda et al. 2009, 2010). Briefly, homogenate from BP and ED samples were filtered using sterile Miracloth (EMD Millipore) and Büchner funnels, and the filtrate was centrifuged at $500 \times g$ for $5 \mathrm{~min}$ at $10^{\circ} \mathrm{C}$. The supernatant was retained and centrifuged at $5,500 \times g$ for $20 \mathrm{~min}$ at $10^{\circ} \mathrm{C}$, after which the supernatant was discarded and the resulting pellet resuspended in $250 \mathrm{ml}$ of BCE and centrifuged again at $10,000 \times g$ for $10 \mathrm{~min}$ at $10^{\circ} \mathrm{C}$. This centrifugation step was repeated two more times (resuspend pellet in $250 \mathrm{ml}$ of BCE and centrifuge at 10,000 $\times g$ for

\section{Surface sterilize, Slice along midrib, Process $1 / 2$ of each leaf in either Slice samples into $~ 1-2 \mathrm{~mm}$ strips, Immerse in enzyme solution enzyme digestion or blender processing

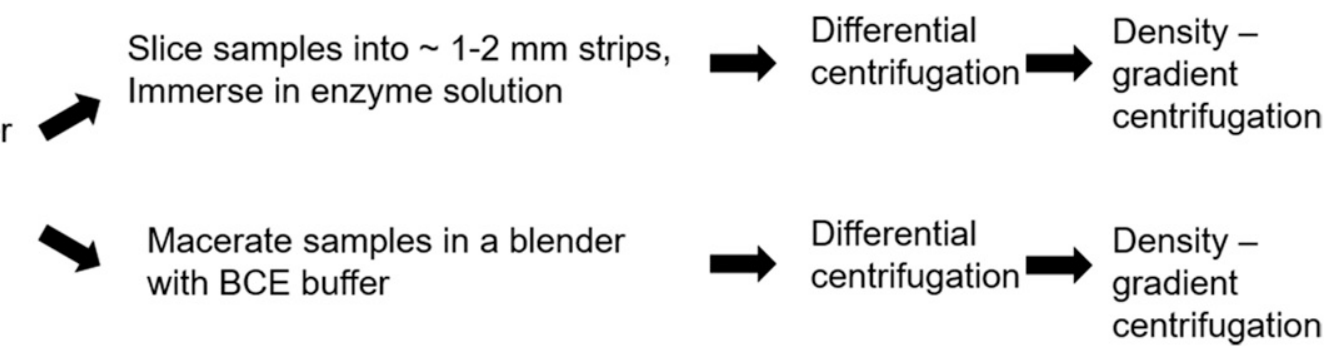

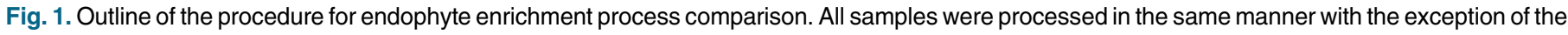
endophyte enrichment process employed for each leaf half. 
$10 \mathrm{~min}$ at $\left.10^{\circ} \mathrm{C}\right)$. The final pellet was resuspended in $6 \mathrm{ml}$ of Tris buffer (50 mM, pH 7.5), carefully layered over $4 \mathrm{ml}$ of Nycodenz (Accurate Chemical \& Scientific Corp.) solution (8 g of Nycodenz in $10 \mathrm{ml}$ of Tris buffer) in sterile glass centrifuge tubes, and then centrifuged at $10,000 \times g$ for $40 \mathrm{~min}$ at $10^{\circ} \mathrm{C}$. Approximately $1 \mathrm{ml}$ containing bacterial cells was carefully pipetted from the band at the interface of buffer and Nycodenz, transferred to a 2-ml tube and mixed with an equal volume of sterile water, and then centrifuged at $14,000 \times g$ for $5 \mathrm{~min}$. The supernatant was discarded and the pellet was frozen at $-20^{\circ} \mathrm{C}$ until DNA extraction.

The protocol for extraction and purification of genomic DNA was adapted from Wilson (Wilson 2001). Both sodium dodecyl sulfate and cetyl trimethylammonium bromide were used along with a high salt concentration for increased removal of proteins and polysaccharides. The frozen pellet obtained in the previous step was resuspended in $567 \mu \mathrm{l}$ of TE buffer and genomic DNA extraction was performed following the protocol of Wilson (2001). Following ethanol precipitation, the air-dried pellet was resuspended in TE buffer and the DNA was quantified with a Qubit 3.0 Fluorometer (Life Technologies, Grand Island, NY).

PCR amplification of the V4 region of the 16S rRNA gene using indexed primers was performed according to the protocol of Kozich et al. (2013). Amplification was verified by gel electrophoresis, with negative controls (primer only, no template) confirming primers and reagents were free from contamination. The PCRs were sent to a core facility at the University of Kentucky, where the reactions were purified with Agencourt AMPure XP beads (Beckman Coulter Inc., Brea, CA), quantified using a Qubit 3.0 Fluorometer (Life Technologies), and pooled in equimolar amounts with approximately $10 \%$ PhiX DNA before sequencing on the Illumina Miseq platform (dual-barcoded, paired-end reads, $2 \times 250$ flow cell).
Samples are identified in the following analysis according to a number that refers to the brand (arbitrarily labeled as 1-3), and by the method employed for homogenization: ED or BP. Demultiplexed forward and reverse reads (primers and barcodes removed) were processed using the Mothur Miseq SOP pipeline (Mothur version 1.44.0, April 2020) (Schloss et al. 2009). Briefly, paired-end reads were assembled into contigs, sequences were filtered for length, ambiguous bases, and homopolymer regions, then aligned to a SILVA reference alignment of the V4 region (SSU Silva 132). Preclustering to merge highly similar (2 bp or less mismatch) sequences was followed by the removal of chimeras and 16S rRNA sequences derived from mitochondrial and chloroplast DNA. Operational taxonomic units (OTUs) were defined using a cutoff of 0.03 ( $97 \%$ similarity) and taxonomic classification based on RDP reference sequences (version 16, February 2016) was done. The data set was subsequently normalized to 22,461 sequences per sample for further analysis (the number of sequences in the sample with the lowest output), resulting in a total of 202 OTUs across all samples. The raw sequence data for this project has been submitted to NCBI-SRA (https://www.ncbi.nlm.nih.gov/sra) with accession number PRJNA524168.

The resulting data set was analyzed using programs in Mothur (Schloss et al. 2009), Phyloseq package for R (McMurdie and Holmes 2013), and LEfSe (linear discriminate analysis effect size) (Segata et al. 2011). In Mothur, nonmetric multidimensional scaling (NMDS) ordinations of Bray-Curtis dissimilarity measure were calculated, ANOSIM (Clarke 1993) analysis was used to compare bacterial community structure, and indicator species analysis (ISA) (Dufrêne and Legendre 1997) was used to identify individual OTUs strongly associated with sample groups. Additionally, Phyloseq was used to calculate and plot alpha diversity measures in R. The

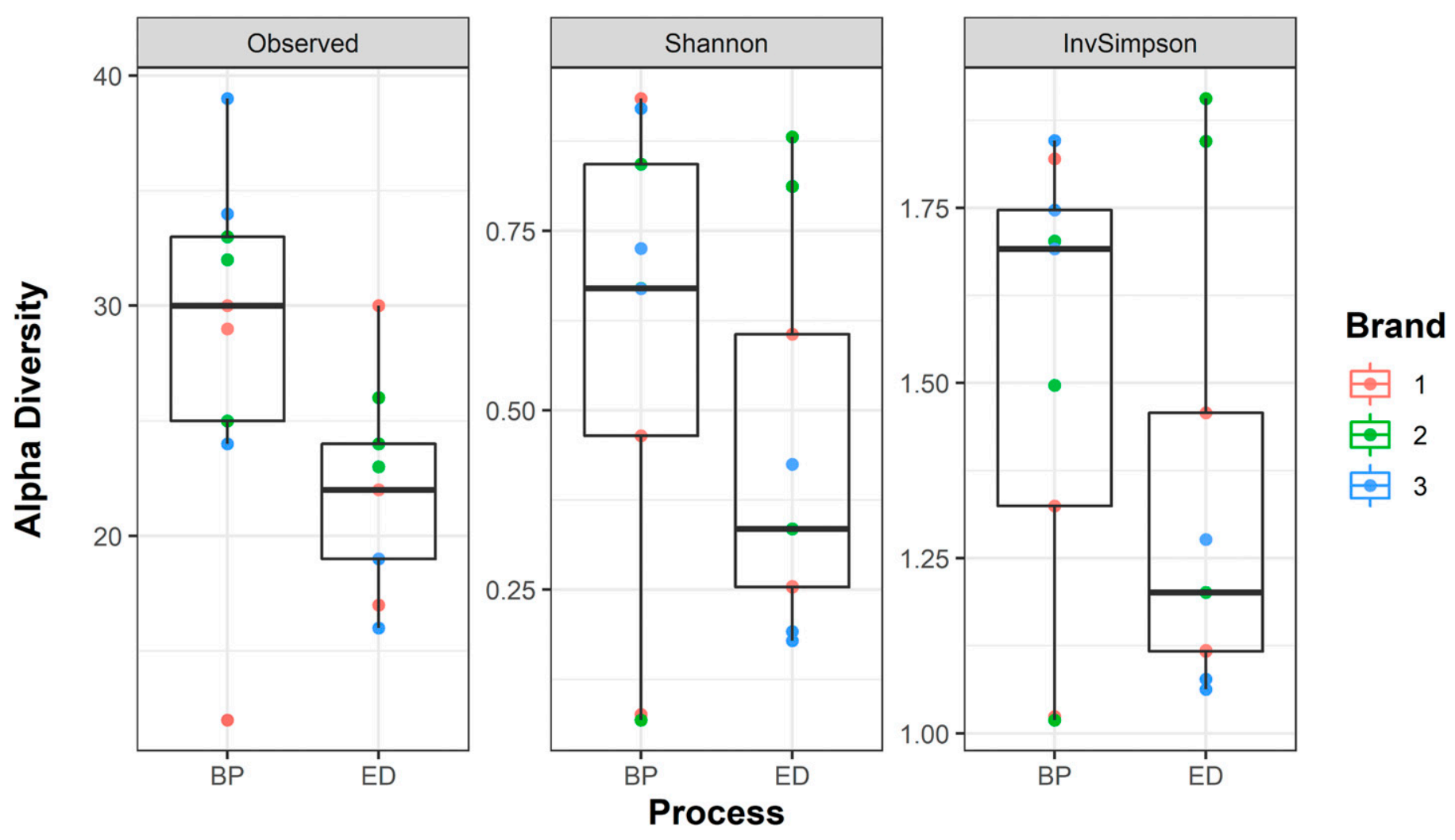

Fig. 2. Alpha diversity measures of samples compared by process; color indicates brand. Boxplot shows the median, with upper and lower hinges at 25th and 75 th percentile, and whiskers at \pm 1.5 the interquartile range. 
program ggplot2 was used in RStudio to plot NMDS ordinations and taxonomy information generated in Mothur (RStudio Team 2015; Wickham 2016).

\section{RESULTS}

After data processing, Good's coverage estimator resulted in values greater than $99 \%$ for all samples, indicating sufficient sampling depth. Alpha diversity measures for richness, Shannon, and inverse Simpson were calculated and compared according to homogenization process, brand, and brand by process using the nonparametric Kruskal-Wallis rank sum test. No significant differences in alpha diversity measures resulted when samples were compared by brand $(P \geq 0.5572)$ or with each brand compared by homogenization process $(P \geq 0.1829)$. When samples were compared by homogenization process, the observed richness was significantly higher for BP samples $(P=0.03051)$, while measures of Shannon and inverse Simpson diversity remained similar $(P \geq$ 0.4529) (Fig. 2). In BP samples, a total of 140 OTUs were observed, whereas the ED samples resulted in 104 OTUs. Of those, there were 97 OTUs in BP samples that did not appear in ED samples, and 63 OTUs that were observed in ED but not BP. Approximately $50 \%$ of OTUs unique to either process were present only once in the data set-the remainder were similarly sparse or were present at only three or less of the samples in that group. Overall, the alpha diversity results indicated that the endophyte communities of all kale samples tested in this study showed low evenness, in which the population was dominated by two high abundance OTUs while the majority of other OTUs were relatively sparse. Total number of OTUs observed is similar to other studies of bacterial leaf endophytes (Puri et al. 2019; Romero et al. 2014; Shi et al. 2014).

NMDS ordination of Bray-Curtis dissimilarity did not reveal strong within-group or between-group membership associations in the data with respect to brand or homogenization process (Fig. 3). ANOSIM of Bray-Curtis distance measures was performed to look for differences in community by brand and homogenization. In this method the $R$ value reflects the effect size of the difference in community, with values closer to 1 indicating a larger difference and values close to 0 indicating less dissimilarity. Comparison by brand gave an $R$ value of $0.098(P=0.14)$, and the homogenization comparison gave an $R$ value of $0.0099(P=0.30)$.

OTUs in the dataset were classified into 11 phyla: Proteobacteria, Bacteroidetes, Firmicutes, Actinobacteria, Verrucomicrobia,
Acidobacteria, Gemmatimonadetes, Planctomycetes, candidate_division_ WPS-1, with 12 OTUs that remained unclassified at the phylum level. In all samples, the dominant Phylum was Proteobacteria ( $>99 \%$ of total phyla in all samples), in which a total of 99 OTUs were classified. Of those, only 26 OTUs had a total abundance of 10 or more in the entire data set; the two most highly abundant OTUs were the only OTUs found to be present in every sample, which classified to the family Enterobacteriaceae and genus Pseudomonas. The top 10 most highly abundant OTUs are all within the phylum Proteobacteria, classify into nine genera, and collectively represent $98.68 \%$ of OTU abundance in the dataset (Fig. 4).

LEfSe analysis found no LDA effect size by brand or process (data not shown), and ISA analysis produced only a small number of OTUs with $P$ values indicating significance $(\alpha=0.05)$. After disregarding those OTUs that were indicated primarily by the abundance of a single sample, three OTUs remained that were indicated by brand, classified as Herbaspirillum, Acinetobacter, and Pseudomonas. Of these, Herbaspirillum was present in all samples of brands 2 and 3 but absent entirely in Brand 1, whereas the Pseudomonas OTU was present only in brand 3, though not in all samples of that brand. The Acinetobacter OTU had the most abundance in brand 2, otherwise this OTU was sparsely present in the dataset.

\section{DISCUSSION}

While little is known about the microbes associated with fieldgrown kale, studies of other leafy greens indicate that plantassociated microbial communities develop from both preharvest and postharvest contact, with higher richness and diversity observed on leaf surfaces in comparison with the leaf endosphere (Jackson et al. 2015). Community profiles here are consistent with previous studies of endophytic communities from leafy greens (Card et al. 2015). Members of the genus Pseudomonas and the family Enterobacteriaceae have been identified as leaf endophytes in a variety of plants, including Brassica species other than kale, leafy greens, and salad vegetables (Card et al. 2015; Hou et al. 2013; Jackson et al. 2013; Zakria et al. 2008). Pseudomonads are well known as plant endophytes, most commonly studied for their plantbeneficial effects such as plant growth promotion, siderophore production, and disease resistance (Card et al. 2015). Nonetheless, the genus does harbor pathogens of plants (e.g., P. syringae) and animals (e.g., P. aeruginosa), as well as organisms implicated in spoilage of fresh fruits and vegetables (Raposo et al. 2017).
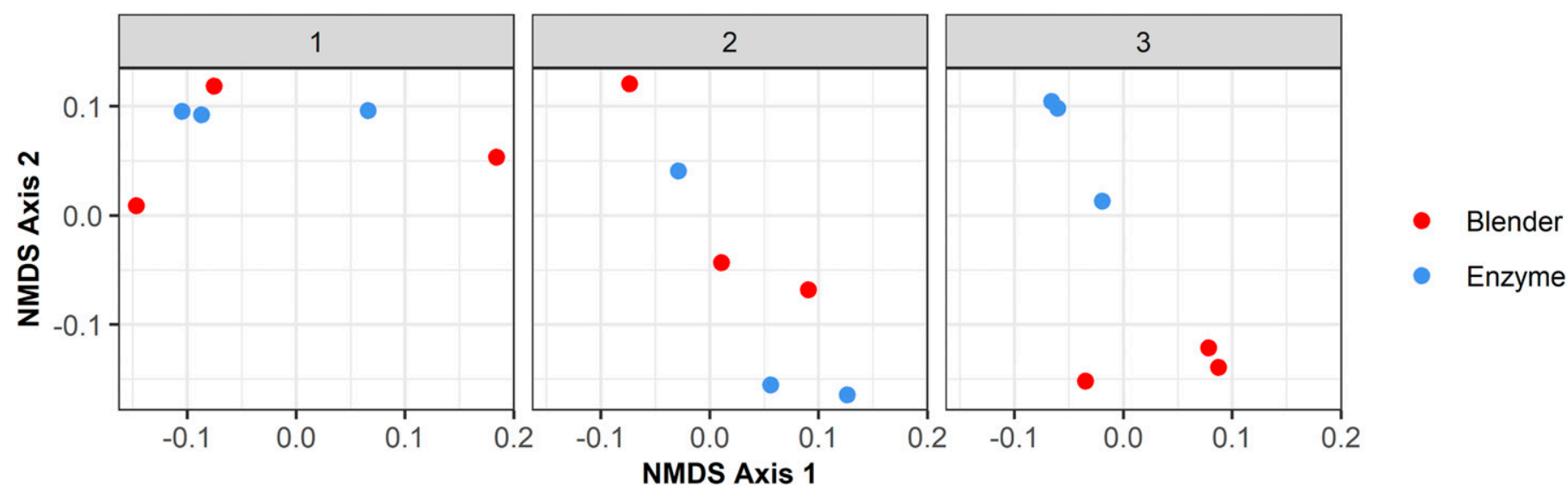

Fig. 3. Nonmetric multidimensional scaling (NMDS) ordination of Bray-Curtis dissimilarity measures of each brand of kale by process of endophyte enrichment. 
While the family Enterobacteriaceae contains several wellknown human and plant pathogens, it is a large and diverse family that is ubiquitous in soil environments and also contains plant-beneficial species that have been implicated in nitrogenfixation, nitrogenase activity, and plant growth promotion (Card et al. 2015; Hinton and Bacon 1995; Octavia and Lan 2014). The Enterobacteriaceae OTU that comprised almost $90 \%$ of the total kale microbiome cannot be further described without additional classification. It should be noted, however, that low levels of several Enterobacteriaceae OTUs classified as Escherichia/Shigella were present in all samples. The extent to which these endophytes pose a threat to human health is not known, though there is increasing evidence that human pathogenic Enterobacteria living as plant leaf endophytes may be more common than previously thought (McNees et al. 2019; Olaimat and Holley 2012). Further work will be necessary to establish whether kale endophytes result primarily from pre- or postharvest colonization, as changes in the microbial communities associated with fresh produce has been shown to change based on postharvest treatment (Jackson et al. 2015). While we have not focused on fungal endophytes here, it is worth noting that fungi are commonly found as leaf endophytes and their community makeup is increasingly being studied using high-throughput sequencing of DNA extracted from plant tissue (Nilsson et al. 2019). Both methodologies outlined here should provide DNA suitable for analysis of mycobiome community structure.

In comparing the BP versus ED methods of homogenizing leaf tissue, BP samples were significantly higher for observed richness than the ED samples; however, the additional OTUs observed were either sparse or singletons. Further analysis using beta diversity, ISA, and LEfSe did not reveal strong differences in community structure and OTU membership by processing method. The higher observed richness in the BP samples may be the result of more complete homogenization; nonetheless, the results of this study indicate that either method is appropriate for endophyte analysis, revealing no exclusion or bias toward any of the observed phylogenetic groups. Brand differences in kale samples were also minimal.

The blender processed samples had a greater percentage of sequences classified as chloroplast/mitochondria than the enzyme digested samples $(22.90 \% \pm 26.32$ versus $4.03 \% \pm 7.47$, respectively); however, the average of the total bacterial sequences from each group after filtering were similar (51,952 for BP and 53,227 for ED), indicating that increased plant DNA from blender processed samples did not significantly affect the extraction and amplification of the microbial DNA. Thus, while blender processing provided a higher observed richness, enzyme digestion resulted in significantly less chloroplast/mitochondrial $16 \mathrm{~S}$ rRNA gene amplicons in the resulting dataset. We speculate that the more gentle approach of digesting plant cell walls resulted in less plant DNA being released into solution, making the differential centrifugation and density separation more efficient. Differential centrifugation and density separation are some of the more time consuming steps of the endophyte enrichment protocol, so it is possible that enzyme digestion can allow for the reduction of some of these steps in order to shorten the protocol and process more samples at a time. While the throughput potential for either of these methods would depend on the available equipment of the researcher, we were able to double the amount of samples processed in 1 day from 12 to $24 \mathrm{~h}$ using the ED method, mainly due to eliminating the need to clean and sterilize blending equipment between samples.
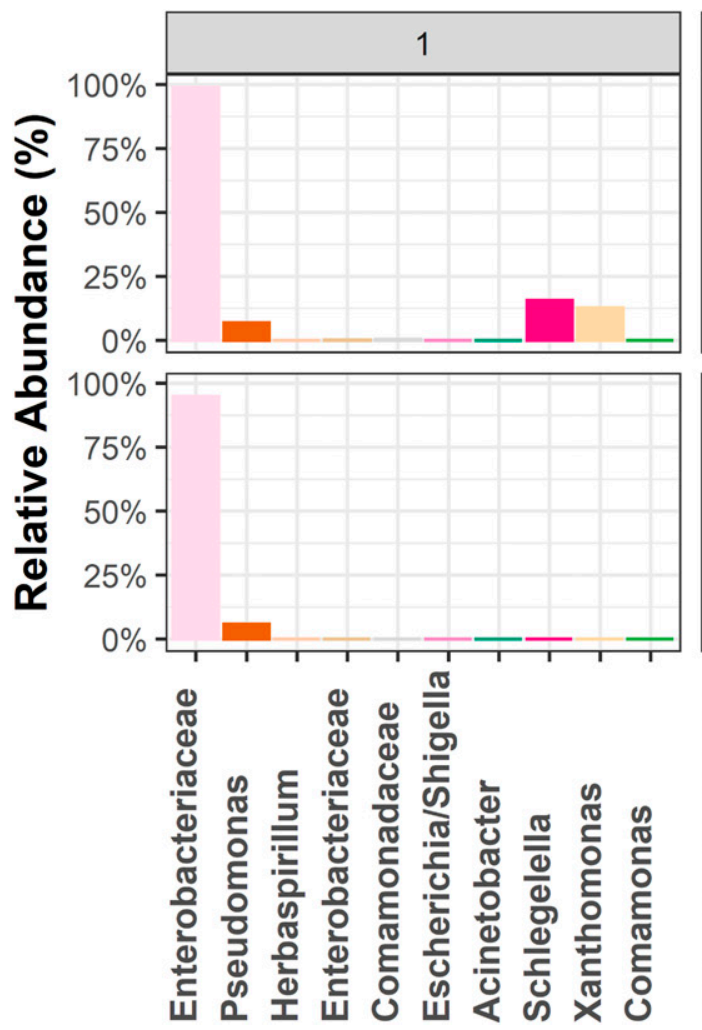
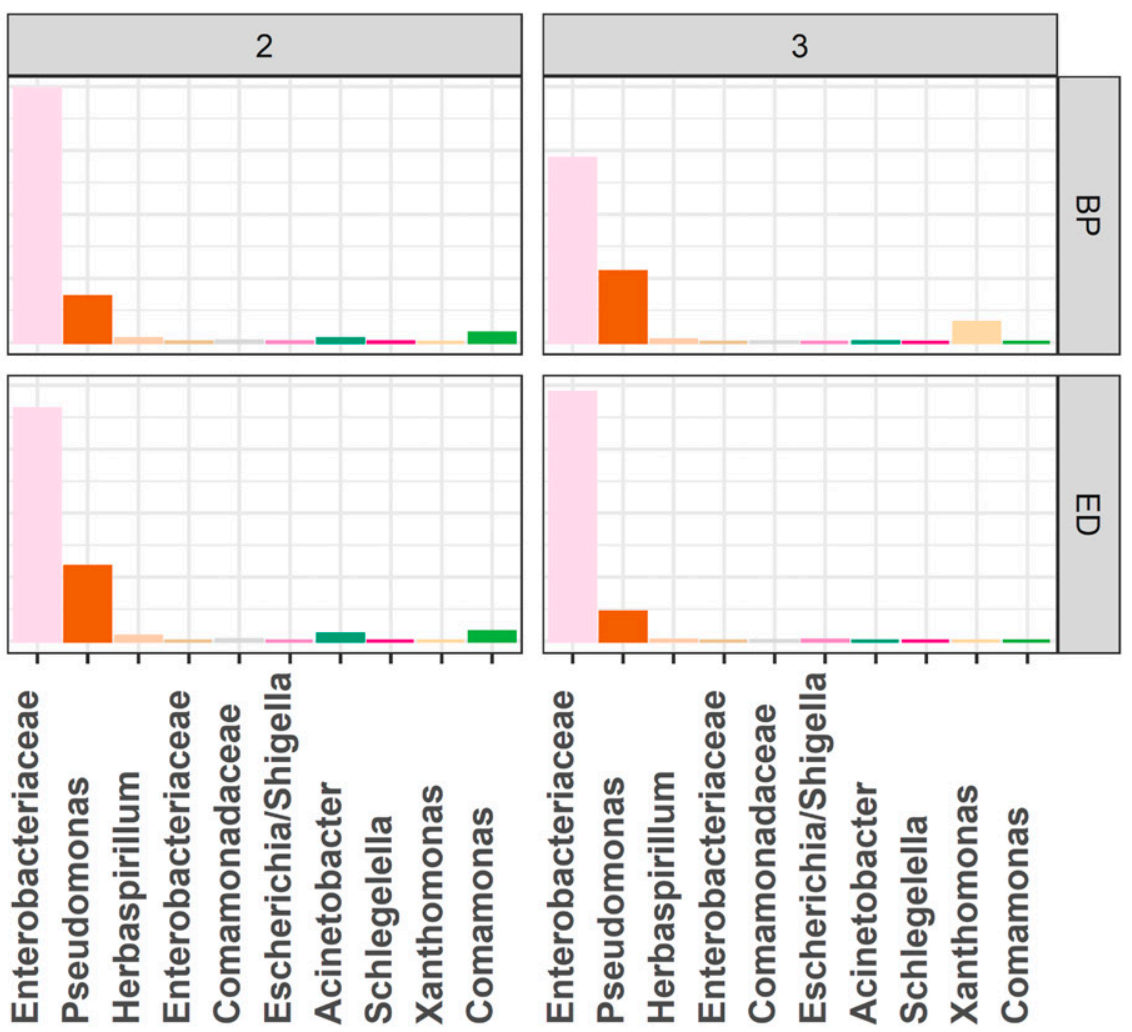

Fig. 4. Relative abundance (summed across replicates) of the 10 most highly abundant operational taxonomic units, which represent over $98 \%$ of the total abundance in the data set. BP, blender processing; and ED, enzyme digestion. 


\section{LITERATURE CITED}

Beckers, B., Op De Beeck, M., Thijs, S., Truyens, S., Weyens, N., Boerjan, W., and Vangronsveld, J. 2016. Performance of 16s rDNA primer pairs in the study of rhizosphere and endosphere bacterial microbiomes in metabarcoding studies. Front. Microbiol. 7:650.

Brandl, M. T. 2006. Fitness of human enteric pathogens on plants and implications for food safety. Annu. Rev. Phytopathol. 44:367-392.

Card, S. D., Hume, D. E., Roodi, D., McGill, C. R., Millner, J. P., and Johnson, R. D. 2015. Beneficial endophytic microorganisms of Brassica-A review. Biol. Control 90:102-112.

Clarke, K. 1993. Nonparametric multivariate analyses of changes in community structure. Aust. J. Ecol. 18:117-143.

Dufrêne, M., and Legendre, P. 1997. Species assemblages and indicator species: The need for a flexible asymmetrical approach. Ecol. Monogr. 67:345-366.

Franco, C., Michelsen, P., Percy, N., Conn, V., Listiana, E., Moll, S., Loria, R., and Coombs, J. 2007. Actinobacterial endophytes for improved crop performance. Australas. Plant Pathol. 36:524-531.

Glick, B. R. 2014. Bacteria with ACC deaminase can promote plant growth and help to feed the world. Microbiol. Res. 169:30-39.

Hinton, D. M., and Bacon, C. W. 1995. Enterobacter cloacae is an endophytic symbiont of corn. Mycopathologia 129:117-125.

Hou, Z., Fink, R. C., Radtke, C., Sadowsky, M. J., and Diez-Gonzalez, F. 2013. Incidence of naturally internalized bacteria in lettuce leaves. Int. J. Food Microbiol. 162:260-265.

Ikeda, S., Kaneko, T., Okubo, T., Rallos, L. E. E., Eda, S., Mitsui, H., Sato, S., Nakamura, Y., Tabata, S., and Minamisawa, K. 2009. Development of a bacterial cell enrichment method and its application to the community analysis in soybean stems. Microbiol. Ecol. 58:703-714.

Ikeda, S., Okubo, T., Anda, M., Nakashita, H., Yasuda, M., Sato, S., Kaneko, T., Tabata, S., Eda, S., Momiyama, A., Terasawa, K., Mitsui, H., and Minamisawa, K. 2010. Community- and genome-based views of plantassociated bacteria: Plant-bacterial interactions in soybean and rice. Plant Cell Physiol. 51:1398-1410.

Ikenaga, M., and Sakai, M. 2014. Application of locked nucleic acid (LNA) oligonucleotide-PCR clamping technique to selectively PCR amplify the SSU rRNA genes of bacteria in investigating the plant-associated community structures. Microbes Environ. 29:286-295.

Jackson, C., Stone, B., and Tyler, H. 2015. Emerging perspectives on the natural microbiome of fresh produce vegetables. Agriculture 5:170-187.

Jackson, C. R., Randolph, K. C., Osborn, S. L., and Tyler, H. L. 2013. Culture dependent and independent analysis of bacterial communities associated with commercial salad leaf vegetables. BMC Microbiol. 13:274

Jiao, J. Y., Wang, H. X., Zeng, Y., and Shen, Y. M. 2006. Enrichment for microbes living in association with plant tissues. J. Appl. Microbiol. 100: 830-837.

Kozich, J. J., Westcott, S. L., Baxter, N. T., Highlander, S. K., and Schloss, P. D. 2013. Development of a dual-index sequencing strategy and curation pipeline for analyzing amplicon sequence data on the MiSeq Illumina sequencing platform. Appl. Environ. Microbiol. 79:5112-5120.

Law, A. D., Fisher, C., Jack, A., and Moe, L. A. 2016. Tobacco, microbes, and carcinogens: Correlation between tobacco cure conditions, tobacco-specific nitrosamine content, and cured leaf microbial community. Microb. Ecol. 72: 120-129.

Maropola, M. K., Ramond, J. B., and Trindade, M. 2015. Impact of metagenomic DNA extraction procedures on the identifiable endophytic bacterial diversity in Sorghum bicolor (L. Moench). J. Microbiol. Methods 112:104-117.

McMurdie, P. J., and Holmes, S. 2013. phyloseq: An R Package for reproducible interactive analysis and graphics of microbiome census data. PLoS One 8: e61217.

McNees, C. R., Greenhut, I. V., Law, A. D., Saleem, M., and Moe, L. A. 2019. Life Within the Leaf: Ecology and Applications of Foliar Bacterial Endophytes. Pages 208-231 in: Endophytes for a Growing World. B. R. Murphy, F. M. Doohan, M. J. Saunders, and T. R. Hodkinson, eds. Cambridge University Press, Cambridge, U.K.
Nilsson, R. H., Anslan, S., Bahram, M., Wurzbacher, C., Baldrian, P., and Tedersoo, L. 2019. Mycobiome diversity: high-throughput sequencing and identification of fungi. Nat. Rev. Microbiol. 17:95-109.

Octavia, S., and Lan, R. 2014. The family Enterobacteriaceae. Pages 225-286 in: The Prokaryotes. E. Rosenberg, E. F. DeLong, S. Lory, E. Stackebrandt, and F. Thompson, eds. Springer, Berlin, Heidelberg.

Olaimat, A. N., and Holley, R. A. 2012. Factors influencing the microbial safety of fresh produce: A review. Food Microbiol. 32:1-19.

Prior, R., Gorges, K., Yurkov, A., and Begerow, D. 2014. New isolation method for endophytes based on enzyme digestion. Mycol. Prog. 13:849-856.

Puri, R. R., Adachi, F., Omichi, M., Saeki, Y., Yamamoto, A., Hayashi, S., Ali, M. A., and Itoh, K. 2019. Metagenomic study of endophytic bacterial community of sweet potato (Ipomoea batatas) cultivated in different soil and climatic conditions. World J. Microbiol. Biotechnol. 35:176.

Raposo, A., Pérez, E., de Faria, C. T., Ferrús, M. A., and Carrascosa, C. 2017. Food spoilage by Pseudomonas spp.-An overview. In: Foodborne Pathogens and Antibiotic Resistance. O. V. Singh, ed. John Wiley \& Sons, New York.

Romero, F. M., Marina, M., and Pieckenstain, F. L. 2014. The communities of tomato (Solanum lycopersicum L.) leaf endophytic bacteria, analyzed by 16S-ribosomal RNA gene pyrosequencing. FEMS Microbiol. Lett. 351: 187-194.

RStudio Team. 2015. RStudio: Integrated Development for R. RStudio, Inc., Boston, MA. https://www.rstudio.com/

Ryan, R. P., Germaine, K., Franks, A., Ryan, D. J., and Dowling, D. N. 2008. Bacterial endophytes: recent developments and applications. FEMS Microbiol. Lett. 278:1-9.

Sakai, M., and Ikenaga, M. 2013. Application of peptide nucleic acid (PNA)-PCR clamping technique to investigate the community structures of rhizobacteria associated with plant roots. J. Microbiol. Methods 92: 281-288.

Schloss, P. D., Westcott, S. L., Ryabin, T., Hall, J. R., Hartmann, M., Hollister, E. B., Lesniewski, R. A., Oakley, B. B., Parks, D. H., Robinson, C. J., Sahl, J. W., Stres, B., Thallinger, G. G., Van Horn, D. J., and Weber, C. F. 2009. Introducing mothur: Open-source, platform-independent, communitysupported software for describing and comparing microbial communities. Appl. Environ. Microbiol. 75:7537-7541.

Segata, N., Izard, J., Waldron, L., Gevers, D., Miropolsky, L., Garrett, W. S., and Huttenhower, C. 2011. Metagenomic biomarker discovery and explanation. Genome Biol. 12:R60.

Sergeant, M. J., Constantinidou, C., Cogan, T., Penn, C. W., and Pallen, M. J. 2012. High-throughput sequencing of 16S rRNA gene amplicons: Effects of extraction procedure, primer length and annealing temperature. PLoS One 7: e38094.

Shi, Y., Yang, H., Zhang, T., Sun, J., and Lou, K. 2014. Illumina-based analysis of endophytic bacterial diversity and space-time dynamics in sugar beet on the north slope of Tianshan mountain. Appl. Microbiol. Biotechnol. 98: 6375-6385.

Sun, L., Qiu, F., Zhang, X., Dai, X., Dong, X., and Song, W. 2008. Endophytic bacterial diversity in rice (Oryza sativa $\mathrm{L}$.) roots estimated by $16 \mathrm{~S}$ rDNA sequence analysis. Microbiol. Ecol. 55:415-424.

Teplitski, M., Barak, J. D., and Schneider, K. R. 2009. Human enteric pathogens in produce: Un-answered ecological questions with direct implications for food safety. Curr. Opin. Biotechnol. 20:166-171.

Tyler, H. L., and Triplett, E. W. 2008. Plants as a habitat for beneficial and/or human pathogenic bacteria. Annu. Rev. Phytopathol. 46:53-73.

Wang, H. X., Geng, Z. L., Zeng, Y., and Shen, Y. M. 2008. Enriching plant microbiota for a metagenomic library construction. Environ. Microbiol. 10: 2684-2691.

Wickham, H. 2016. ggplot2: Elegant Graphics for Data Analysis. SpringerVerlag, New York.

Wilson, K. 2001. Preparation of genomic DNA from bacteria. Curr. Protoc. Mol. Biol. 56:2.4.1-2.4.5

Zakria, M., Ohsako, A., Saeki, Y., Yamamoto, A., and Akao, S. 2008. Colonization and growth promotion characteristics of Enterobacter sp. and Herbaspirillum sp. on Brassica oleracea. Soil Sci. Plant Nutr. 54: 507-516. 